\title{
Fermilab Booster Operational Status: Beam Loss and Collimation*
}

\author{
Robert C. Webber ${ }^{\dagger}$ \\ Fermi National Accelerator Laboratory, Batavia, IL 60510, USA
}

\begin{abstract}
Beam loss reduction and control challenges confronting the Fermilab Booster are presented in the context of the current operational status. In Summer 2002 the programmatic demand for $8 \mathrm{GeV}$ protons will increase to 5E20/year. This is an order of magnitude above recent high rates and nearly as many protons as the machine has produced in its entire 30-year lifetime. Catastrophic radiation damage to accelerator components must be avoided, maintenance in an elevated residual radiation environment must be addressed, and operation within a tight safety envelope must be conducted to limit prompt radiation in the buildings and grounds around the Booster. Diagnostic and performance tracking improvements, enhanced orbit control, and a beam loss collimation/localization system are essential elements in the approach to achieving the expected level of performance and are described here.
\end{abstract}

\section{INTRODUCTION}

Efforts are underway to meet forthcoming demands on the Fermilab Booster. In addition to supplying 1 E16 protons per hour (pph) for antiproton production for Collider Run II, Booster is requested to provide up to $9 \mathrm{E} 16 \mathrm{pph}$ for the MiniBooNE experiment [1][2]. The highest historical beam rate was about 2E16 pph, under much less restrictive radiation constraints than apply today. Booster currently operates near the 5E12 protons per pulse (ppp) expected by each user. The increased hourly beam throughput will be achieved by increasing average beam pulse rates to approximately $6 \mathrm{~Hz}$. Recently Booster has provided beam at average rates less than $1 \mathrm{~Hz}$. Reduction and control of beam loss and resulting radiation impacts is essential.

\section{RECENT OPERATIONS}

The Booster has operated for the past year in support of Tevatron Collider Run II operations and Recycler Ring commissioning. Figure 1 shows operation for the first 14 weeks of 2002 at typical peak pulse intensities of about 4.5E12 ppp and peak rates of $1.2 \mathrm{E} 14$ protons per minute $(7.2 \mathrm{E} 15 \mathrm{pph})$. These rates are typically sustained for 12-16 hours per day for antiproton production with much lighter demands the remainder of the time. During these 14 weeks, a total of $7.06 \mathrm{E} 18$ protons were accelerated corresponding to an overall time-averaged rate of 3E15 pph.

Several measures of beam loss performance have been recently established or improved to track the effectiveness of machine improvements. More than 50

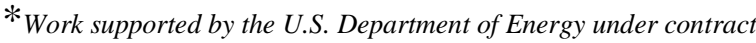
No. DE-AC02-76CH0300O.

†webber@fnal.gov
}

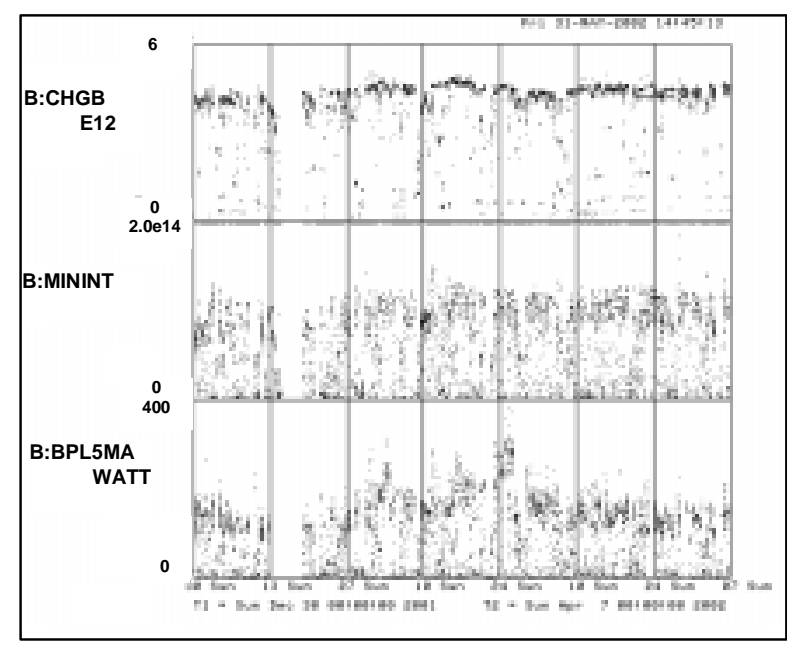

FIGURE 1. Recent 14 weeks of operation.

Top: per pulse intensity. Middle: protons per minute accelerated. Bottom: average beam power lost.

radiation monitors (chipmunks), installed around Booster as radiation safety interlocks, provide useful average beam loss information that is data logged. Performance relative to interlock trip levels is tracked and used to project the potential trouble spots as beam rates are increased. This information is available at http://www-bd.fnal.gov/proton/booster/chipmunks/.

Improvements have been made in the data acquisition and data logging aspects of the Booster Beam Loss Monitor (BLM) system. The beam loss observed by each of $>50$ BLMs during each millisecond of each Booster cycle is measured and individually accumulated for each time interval. The accumulated values and the total accumulated loss are logged to provide long-term capability of tracking performance as a function of time in the cycle at each BLM location. Figure 2 is a typical BLM plot that can 


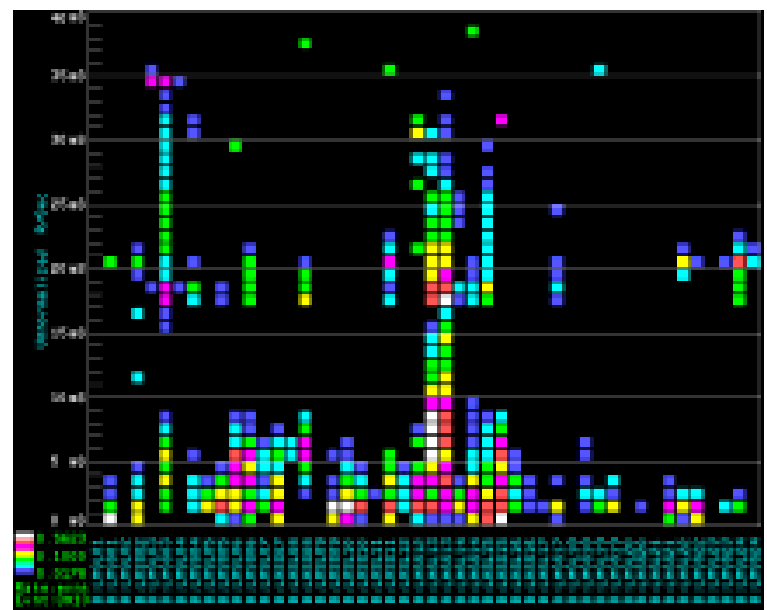

FIGURE 2. Color gradient BLM plot. Horizontal: BLM location. Vertical: time in cycle.

be generated from either current or logged BLM data showing color-coded loss levels around the ring vs. time in the acceleration cycle. The lower horizontal band in Figure 2 shows widely distributed losses during the first $5 \mathrm{msec}$ of the cycle and the band midway up the plot shows losses at transition. The vertical bands near the left and center of the plot are losses due to apertures at the two Booster extraction regions.

A real-time 100-second running average of the total loss at each BLM location is also produced and data logged. These running averages are incorporated into the Booster Alarms and Limits system to alert machine operators and potentially inhibit beam should the loss at any location exceed a predetermined limit. This feature is expected to be the front-line defense in controlling component irradiation and residual radiation levels. Data on current machine performance relative to initial limit settings is currently at http://www-bd.fnal.gov/proton/booster/blms/.

Another real-time measurement of total beam energy lost is obtained from the Booster beam current monitor. That signal is effectively differentiated throughout each machine cycle and weighted by the energy at the time of the loss. The result is integrated throughout each cycle to yield a total beam energy lost value and then accumulated over all cycles to provide a running five-minute time-average of beam power lost. Typical levels of 150 to 250 watts during peak operations are shown in Figure 1. Given the 474-meter Booster circumference this represents an average power loss just less than 0.5 watts/meter.

Each shutdown, typically 2 to 8 hours after beam has been turned off, the radiation level at a fixed set of radiation survey points on or near the Booster beam line is measured and recorded to be compared and correlated with the real-time beam loss measures. Data has been accumulated since June 2001. Figure 3 shows the residual radiation levels for the last 9 months at "normal" long and short straight sections around Booster, i.e. excluding injection, extraction, and RF cavity locations. Figure 4 shows data from the same time period at the Booster RF cavity locations. The RF cavity data is particularly important because the power amplifiers, located directly on top of the cavities, are the highest maintenance items in the tunnel. Note that Figure 3 has a logarithmic vertical scale and that rates are "at contact" on or near the beam pipe, whereas Figure 4 has a linear scale and rates are at "one foot" from the cavities. This data corresponds to operation at a time-averaged beam rate of 3E15 pph.

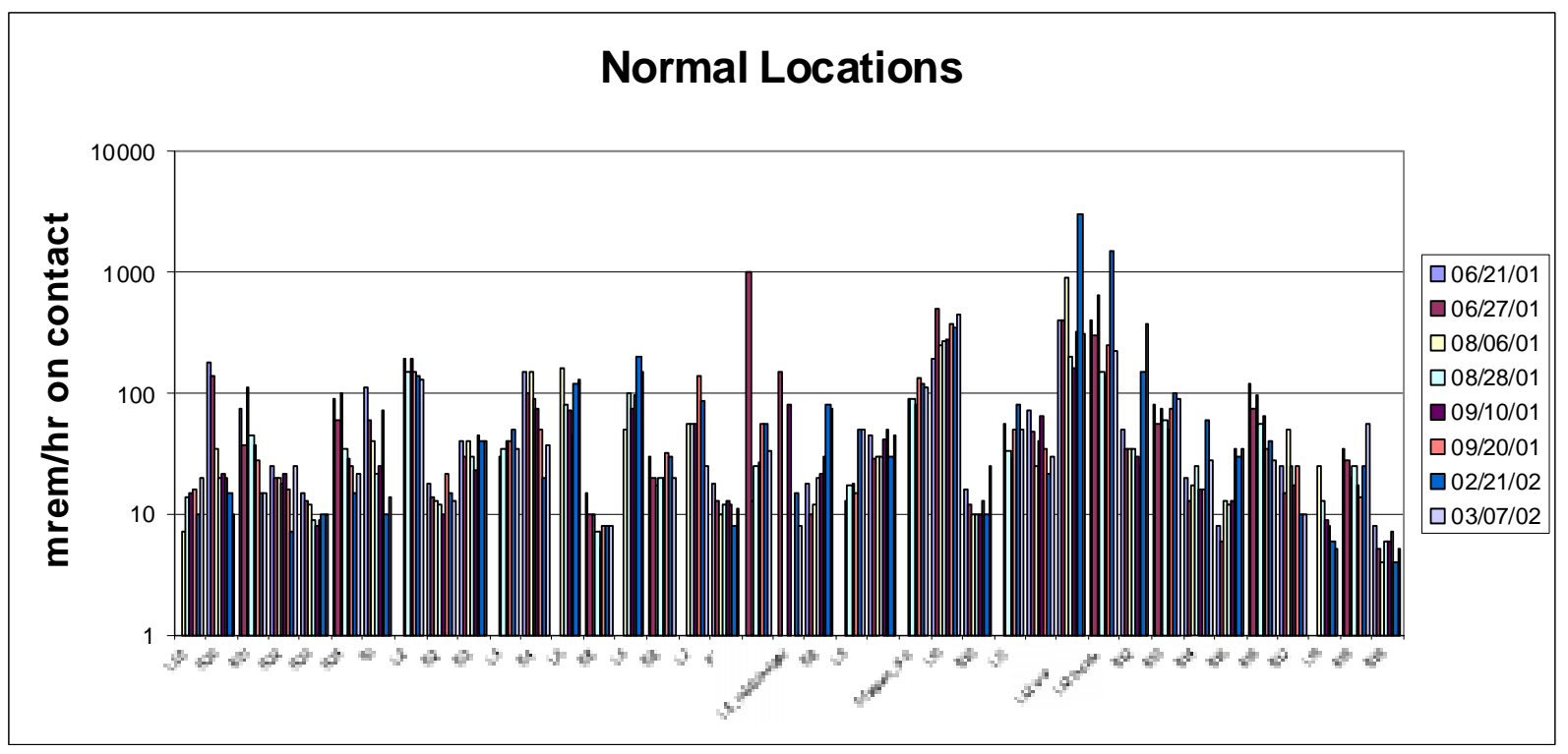

FIGURE 3. Residual radiation levels on contact at "normal" locations around Booster over recent 9 months. 


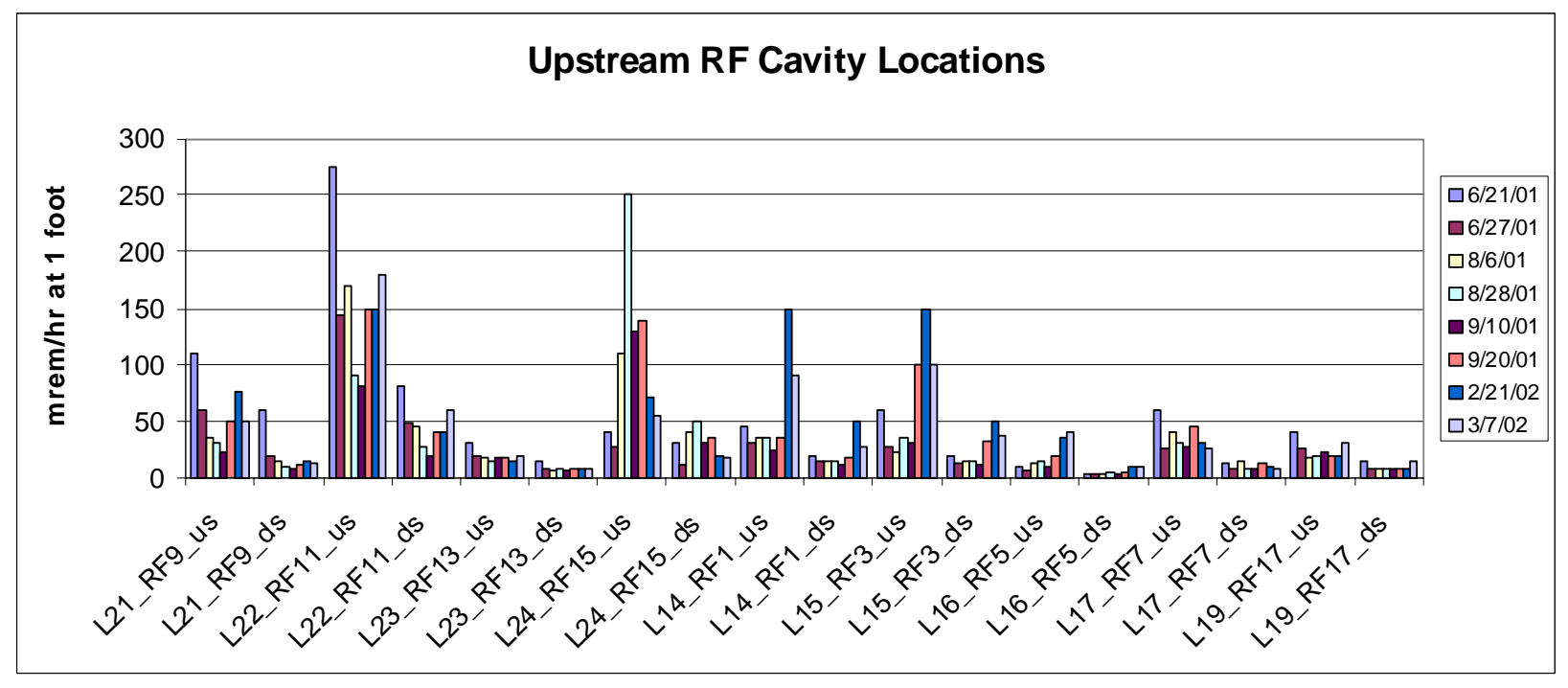

FIGURE 4. Residual radiation levels at one foot at RF cavity locations around Booster over recent 9 months.

\section{IMPROVEMENT ACTIVITIES}

One avenue to reduced beam loss is through improved orbit control with the addition of ramp capability to the Booster dipole corrector magnet system. Figure 5 shows first test results of ramped correctors attempting to hold position fixed throughout the cycle. Operational utility of the ramping capability will rely on soundly and robustly engineered software with a simple and friendly user interface to enforce and facilitate operational discipline.

A beam collimation system has been designed [3] and recently installed in Booster to localize unavoidable beam losses. The system spans two of Booster's 24 lattice periods and includes in each plane a thin carbon primary collimator followed by two 0.6 meter copper energy absorbing secondary collimators. See Figure 6. Initial tests of the collimation system are just beginning and an acceptable collimator shielding design is still to be completed.

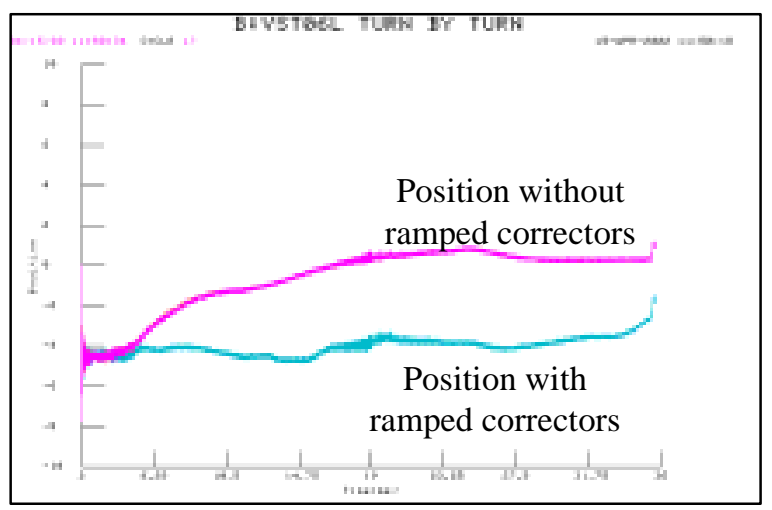

FIGURE 5. Ramped correctors in action.

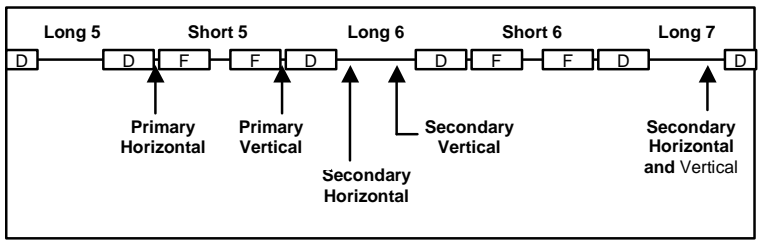

FIGURE 6. Collimator layout.

\section{CONCLUSION}

Booster is still far from achieving the requested $1 \mathrm{E} 17$ pph beam rate. Improved orbit control and clean collimation system operation will be key to improved performance. Operational loss limits must be established to avoid disastrous component radiation damage and unmanageable maintenance problems. Operational discipline with automated loss monitoring and limiting and data logging tools is important. In any case, Booster will face a new era in dealing with operations in a high radiation environment.

\section{REFERENCES}

1. MiniBooNE, http://www-boone.fnal.gov/.

2. R. Webber, "Challenges to the Fermilab Linac and Booster Accelerators", Proceedings of the 2001 Particle Accelerator Conference, IEEE Catalog \# 01CH37268, pp. 2581-2583.

3. A. Drozhdin et al., "Beam Loss, Residual Radiation, and Collimation and Shielding in the Fermilab Booster", Proceedings of the 2001 Particle Accelerator Conference, IEEE Catalog \# 01CH37268, pp. 2569-2571. 